\title{
A MODEL BASED ON FUZZY LINGUISTIC INFORMATION TO EVALUATE THE QUALITY OF DIGITAL LIBRARIES
}

\author{
F. J. CABRERIZO \\ Department of Software Engineering and Computer Systems \\ Distance Learning University of Spain (UNED), Madrid 28040, Spain \\ cabrerizo@issi.uned.es \\ J. LÓPEZ-GIJÓN, A. A. RUÍZ \\ Department of Library Science \\ University of Granada, Granada 18071, Spain \\ \{jgijon,aangel\}@ugr.es \\ E. HERRERA-VIEDMA \\ Department of Computer Science and Artificial Intelligence \\ University of Granada, Granada 18071, Spain \\ viedma@decsai.ugr.es \\ Received (Day Month Year) \\ Revised (Day Month Year)
}

\begin{abstract}
The Web is changing the information access processes and it is one of the most important information media. Thus, the developments on the Web are having a great influence over the developments on others information access instruments as digital libraries. As the development of digital libraries is to satisfy user need, user satisfaction is essential for the success of a digital library. The aim of this paper is to present a model based on fuzzy linguistic information to evaluate the quality of digital libraries. The quality evaluation of digital libraries is defined using users' perceptions on the quality of digital services provided through their Web sites. We assume a fuzzy linguistic modeling to represent the users' perception and apply automatic tools of fuzzy computing with words based on the LOWA and LWA operators to compute global quality evaluations of digital libraries. Additionally, we show an example of application of this model where three Spanish academic digital libraries are evaluated by fifty users.
\end{abstract}

Keywords: Quality; digital libraries; web site; fuzzy linguistic modeling.

\section{Introduction}

The World Wide Web is a popular and interactive medium to collect, disseminate and access an increasingly huge amount of information: it constitutes the basic mainstay of the so called "Information and Knowledge Society". Due to its spectacular growth, related to both Web resources (pages, sites and services) and visitors, the Web is nowadays the main information repository. This explosive growth of 
the World Wide Web stimulates the development of fast and effective automated systems that support an easy and effective access to the information relevant to specific users' needs. ${ }^{1}$ Digital libraries ${ }^{2,3,4,5}$ are one of these automated systems.

Since 1990s, the Internet and the Web has become the primary platform for libraries to build and deliver information resources, services and instructions. Nowadays, in the digital age, we find two kinds of library user information services: ${ }^{3}$

(i) Traditional library user information services, which are based on a face-toface personal communication and are developed on-site, as for example: onsite bibliographic instruction, consultation, user technical support, classroom instruction, and so on.

(ii) Electronic library user information services, which are based on the Web, can be developed on-site or off-site, and are accessible without any geographic and time limitations, as for example: integrated library systems, distance learning services, e-databases services, web library catalogs, open source journal information, web search engines, instant messaging services, virtual reference, etc.

Depending on the library framework, both services are necessary and complementary to develop library activities. However, electronic services allow us to improve the efficiency of the libraries and, therefore, we find hybrid libraries ${ }^{6}$ that keep some traditional services but with a great tendency to create new digital services using all Web possibilities. In this framework, we have to deal with new challenges and key issues if we want to offer quality library services to the users, as for example: ${ }^{3}$ role academic libraries, quality information resources, Web instructions and training, new assessment and evaluation methodologies, etc. Therefore, in last years, the new concept of digital library is growing.

Digital libraries are information collections that have associated services delivered to user communities using a variety of technologies. The information collections can be scientific, business or personal data, and can be represented as digital text, image, audio, video, or other media. This information can be digitalized paper or born digital material and the services offered on such information can be varied, and can be offered to individuals or user communities. Furthermore, digital libraries are components in several types of applications in area such as cultural heritage, health, government, learning, and science. Technological advances in areas like information searching and retrieval, information storage, user interfaces, telecommunications as well as the increasing availability of a variety of multimedia collections make it possible to offer new and better services for user groups.

Internet access has resulted in digital libraries that are increasingly used by diverse communities for diverse purposes, and in which sharing and collaboration have become important social elements. As digital libraries become commonplace, as their contents and services become more varied, people expect more sophisticated services from their digital libraries. ${ }^{7}$

As digital libraries have been around for a few years now, an increasing number of users have some familiarity with them. This emergence of digital libraries calls 
for the need for the evaluation of digital libraries. Furthermore, the expectations and demands for better service and functionality from these users are increasing. Thus, the importance of quality in digital libraries content and services is higher than ever. ${ }^{8}$ In this way, evaluation of digital libraries is an essential component for the design of effective digital libraries. ${ }^{9}$ Evaluation is a research activity, and it has both theoretical and practical impact. ${ }^{10}$ An evaluation is a judgment of worth. The objective of digital libraries evaluation is to assess to what extent a digital library meets its objectives and offer suggestions for improvements. ${ }^{4}$ Even though there are no standard evaluation criteria and evaluation techniques for digital libraries evaluation, digital libraries evaluation research has been conducted on different aspects. Most of the research on evaluation of digital libraries has applied from researchers themselves. However, as digital libraries are designed for users to use, the quality of digital libraries needs be judged by the users of them.

The main of this paper is to present a model based on fuzzy linguistic information to evaluate the quality of digital libraries. This evaluation model presents a set of subjective criteria related to the Web sites of digital libraries and a computation instrument of quality assessments. We assume that the quality of a digital library is measured through users' perceptions on the digital services offered through its Web site. Users are invited to fill in a survey built on the set of subjective criteria. To measure quality, conventional measurement tools used by the customers are devised on cardinal or ordinal scales. However, the scores do not necessarily represent user preference. This is because respondents have to internally convert preference to scores and the conversion may introduce distortion of the preference. ${ }^{11}$ For this reason, we use an ordinal fuzzy linguistic modeling ${ }^{12}$ to represent the users' perceptions and tools of computing with words based on the linguistic aggregation operators LOWA ${ }^{12}$ and $\mathrm{LWA}^{13}$ to compute the quality assessments.

The rest of the paper is set out as follows. In Sec. 2, the ordinal fuzzy linguistic modeling for computing with words is discussed. Section 3 describes the model based on fuzzy linguistic information to evaluate the quality of digital libraries. Section 4 presents an example of application of this model where three Spanish academic digital libraries are evaluated by fifty users. A discussion on the proposed evaluation model is presented in Sec. 5. Finally, some concluding remarks are pointed out in Sec. 6 .

\section{A Fuzzy Linguistic Approach for Computing with Words}

Many problems present fuzzy and vague qualitative aspects (decision making, ${ }^{14,15,16}$ risk assessment,${ }^{17}$ information retrieval, ${ }^{18,19,20,41}$ etc.). In such problems, the information cannot be assessed precisely in a quantitative form, but it may be done in a qualitative one, and thus, the use of a linguistic approach is necessary. The fuzzy linguistic approach is an approximate technique appropriate to deal with fuzzy and vague qualitative aspects of problems. ${ }^{18,41,22}$ It models linguistic information by means of linguistic terms supported by linguistic variables. ${ }^{23,24,25}$ These are vari- 
ables whose values are not numbers but words or sentences in a natural or artificial language. A linguistic variable is defined by means of a syntactic rule and a semantic rule. The fuzzy linguistic approach is less precise than the numerical one, but, however, it presents the following advantages:

(i) The linguistic description is easily understood by human beings even when the concepts are abstract or the context is changing.

(ii) It diminishes the effects of noise since, as it is known, the more refined assessment scale is, then more sensitive to noise (linguistic scales are less refined than numerical scales and consequently they are less sensitive to error apparition and propagation).

The ordinal fuzzy linguistic approach ${ }^{12,13}$ is a very useful kind of fuzzy linguistic approach used for modeling the computing with words process as well as linguistic aspects of problems. It facilitates the fuzzy linguistic modeling very much because it simplifies the definition of the semantic and syntactic rules. It is defined by considering a finite and totally ordered label set $S=\left\{s_{i}\right\}, i \in\{0, \ldots, \mathcal{T}\}$ in the usual sense, i.e., $s_{i} \geq s_{j}$ if $i \geq j$, and with odd cardinality. Typical values of cardinality used in the linguistic models are odd values, such as 7 or 9 , with an upper limit of granularity of 11 or no more than 13, where the mid term represents an assessment of "approximately 0.5 ", and the rest of the terms being placed symmetrically around it. These classical values seems to fall in line Miller's observation about the fact that human beings can reasonably manage to bear in mind seven or so items. ${ }^{26}$ The semantics of the linguistic term set is established from the ordered structure of the label set by considering that each linguistic term for the pair $\left(s_{i}, s_{\mathcal{T}-i}\right)$ is equally informative. For example, we can use the following set of nine labels to provide the user evaluations: $\{N=$ None, $E L=$ Extremely Low, $V L=$ Very Low, $L=$ Low, $M=$ Medium, $H=$ High,$V H=$ Very High, EH = Extremely High, $T=$ Total\}.

In any linguistic approach we need management operators of linguistic information. ${ }^{12,13}$ An advantage of the ordinal fuzzy linguistic approach is the simplicity and quickness of its computational model. It is based on the symbolic computation ${ }^{12,13}$ and acts by direct computation on labels by taking into account the order of such linguistic assessments in the ordered structure of linguistic terms. This symbolic tool seems natural when using the fuzzy linguistic approach, because the linguistic assessments are simply approximations which are given and handled when it is impossible or unnecessary to obtain more accurate values. Thus, in this case, the use of membership functions associated to the linguistic terms is unnecessary.

Usually, the ordinal fuzzy linguistic model for computing with words is defined by establishing (i) a negation operator, (ii) comparison operators based on the ordered structure of linguistic terms, and (iii) adequate aggregation operators of ordinal fuzzy linguistic information. In most ordinal fuzzy linguistic approaches the negation operator is defined from the semantics associated to the linguistic terms 
as $N e g\left(s_{i}\right)=s_{j} \mid j=\mathcal{T}-i$; and there are defined two comparison operators of linguistic terms:

- Maximization operator: $\operatorname{MAX}\left(s_{i}, s_{j}\right)=s_{i}$ if $s_{i} \geq s_{j}$.

- Minimization operator: $M I N\left(s_{i}, s_{j}\right)=s_{i}$ if $s_{i} \leq s_{j}$.

In the following subsections, we present two aggregation operators based on symbolic computation to complete the ordinal linguistic computational model.

\subsection{The LOWA operator}

An important aggregation operator of ordinal linguistic values based on symbolic computation is the LOWA operator. ${ }^{12}$ The Linguistic Ordered Weighted Averaging (LOWA) is an operator used to aggregate non-weighted ordinal linguistic information, i.e., linguistic information values with equal importance. ${ }^{12}$

Definition 2.1. Let $A=\left\{a_{1}, \ldots, a_{m}\right\}$ be a set of labels to be aggregated, then the LOWA operator, $\phi$, is defined as:

$$
\begin{aligned}
& \phi\left(a_{1}, \ldots, a_{m}\right)=W \cdot B^{T}=\mathcal{C}^{m}\left\{w_{k}, b_{k}, k=1, \ldots, m\right\} \\
& =w_{1} \odot b_{1} \oplus\left(1-w_{1}\right) \odot \mathcal{C}^{m-1}\left\{\beta_{h}, b_{h}, h=2, \ldots, m\right\},
\end{aligned}
$$

where $W=\left[w_{1}, \ldots, w_{m}\right]$ is a weighting vector, such that, $w_{i} \in[0,1]$ and $\Sigma_{i} w_{i}=1$. $\beta_{h}=w_{h} / \Sigma_{2}^{m} w_{k}$, and $B=\left\{b_{1}, \ldots, b_{m}\right\}$ is a vector associated to $A$, such that, $B=\sigma(A)=\left\{a_{\sigma(1)}, \ldots, a_{\sigma(m)}\right\}$, where, $a_{\sigma(j)} \leq a_{\sigma(i)} \forall i \leq j$, with $\sigma$ being a permutation over the set of labels $A$. $\mathcal{C}^{m}$ is the convex combination operator of $m$ labels and if $m=2$, then it is defined as:

$$
\mathcal{C}^{2}\left\{w_{i}, b_{i}, i=1,2\right\}=w_{1} \odot s_{j} \oplus\left(1-w_{1}\right) \odot s_{i}=s_{k},
$$

such that, $k=\min \left\{\mathcal{T}, i+\operatorname{round}\left(w_{1} \cdot(j-i)\right)\right\}, s_{j}, s_{i} \in S,(j \geq i)$, where "round" is the usual round operation, and $b_{1}=s_{j}, b_{2}=s_{i}$. If $w_{j}=1$ and $w_{i}=0$, with $i \neq$ $j \forall i$, then the convex combination is defined as: $\mathcal{C}^{m}\left\{w_{i}, b_{i}, i=1, \ldots, m\right\}=b_{j}$.

The LOWA operator is an "or-and" operator ${ }^{12}$ and its behavior can be controlled by means of $W$. In order to classify OWA operators with regards to their localization between "or" and "and", Yager ${ }^{27}$ introduced a measure of orness, associated with any vector $W: \operatorname{orness}(W)=\frac{1}{m-1} \sum_{i=1}^{m}(m-i) w_{i}$. This measure characterizes the degree to which the aggregation is like an "or" (MAX) operation. Note that an OWA operator with orness $(W) \geq 0.5$ will be an orlike, and with orness $(W)<0.5$ will be an andlike operator.

An important question of the LOWA operator is the determination of the weighting vector $W$. In Ref. 24, it was defined an expression to obtain $W$ that allows to represent the concept of fuzzy majority ${ }^{28}$ by means of a fuzzy linguistic nondecreasing quantifier $Q:{ }^{29}$

$$
w_{i}=Q(i / n)-Q((i-1) / n), i=1, \ldots, n .
$$

When a fuzzy linguistic quantifier $Q$ is used to compute the weights of LOWA operator $\phi$, it is symbolized by $\phi_{Q}$. 


\subsection{The LWA operator}

Another important aggregation operator of ordinal linguistic values is the Linguistic Weighted Averaging (LWA) operator. ${ }^{13}$ It is based on the LOWA operator and is defined to aggregate weighted ordinal fuzzy linguistic information, i.e., linguistic information values with not equal importance.

As it is known, the aggregation of weighted information involves two activities: (i) The transformation of the weighted information under the importance degrees by means of a transformation function $h$, and (ii) the aggregation of the transformed weighted information by means of an aggregation operator of non-weighted information $f$. The transformation function depends upon the type of aggregation of weighted information which is going to be performed. In Ref. 27, Yager discussed the effect of the importance degrees on the "MAX" and "MIN" types of aggregation and suggested a class of functions for importance transformation in both types of aggregation. For the MIN aggregation, he suggested a family of $t$-conorms acting on the weighted information and the negation of the importance degree, which presents the non-increasing monotonic property in these importance degrees. For the MAX aggregation, he suggested a non-decreasing monotonic property in these importance degrees.

Following the above idea, the LWA operator is defined in Ref. 12. Here, we redefine it to simplify its expression using the orness measure and as $f$ the LOWA operator $\phi$.

Definition 2.2. The aggregation of a set of weighted linguistic opinions, $\left\{\left(c_{1}, a_{1}\right), \ldots,\left(c_{m}, a_{m},\right)\right\}, c_{i}, a_{i} \in S$, according to the LWA operator, $\Phi$, is defined as:

$$
\Phi\left[\left(c_{1}, a_{1}\right), \ldots,\left(c_{m}, a_{m}\right)\right]=\phi\left(h\left(c_{1}, a_{1}\right), \ldots, h\left(c_{m}, a_{m}\right)\right),
$$

where $a_{i}$ represents the weighted opinion, $c_{i}$ the importance degree of $a_{i}$, and $h$ is the transformation function defined depending on the weighting vector $W$ used for the LOWA operator $\phi$, such that, $h=\operatorname{MIN}\left(c_{i}, a_{i}\right)$ if orness $(W) \geq 0.5$, and $h=\operatorname{MAX}\left(\operatorname{Neg}\left(c_{i}\right), a_{i}\right)$ if orness $(W)<0.5$.

We should point out that the LOWA and LWA operators are the basis of the new fuzzy linguistic evaluation model of digital libraries that we present in this paper. We have chosen these operators due to the following reasons:

(i) Both operators are complementary (the LWA operator is defined from the LOWA operator) and this simplifies the design of the evaluation model.

(ii) Both operators act by symbolic computation and, therefore, linguistic approximation processes are unnecessary and this simplifies the processes of computing with words.

(iii) The concept of fuzzy majority represented by linguistic quantifiers acts in their processes of computation and, in such a way, the assessments on digital libraries are obtained according to the majority of evaluations provided by the users. 


\section{Evaluating Quality in Digital Libraries}

In this section, we present the model based on fuzzy linguistic information to evaluate the quality of digital libraries. Previously, we review some aspects on evaluation of quality in digital libraries.

\subsection{On evaluation of quality in digital libraries}

Digital libraries are new and innovative information systems, under constant development and change, and, therefore, evaluation is of critical importance to ensure not only their correct evolution but also their acceptance by the user and application communities. The objective of digital libraries evaluation is to assess to what extent a digital library meets its objectives and offer suggestions for improvements. ${ }^{4}$ Digital libraries evaluation has many facets depending on the characteristics and the perspective of the evaluating agent.

Different approaches to evaluate the success of a digital library have been studied $^{8,9,10,31,32,33,34,35,36}$ involving users, collections, and systems, aimed at identifying generalizable metrics or context specific methods. The most recognized digital libraries evaluation criteria are derived from evaluation criteria for traditional libraries, information retrieval system performance and human-computer interaction. ${ }^{4,10,31,32}$ Very few studies actually apply all the digital evaluation criteria to assess a digital library. Many of the studies focus on the evaluation of usability of digital libraries. After reviewing usability tests in selected academic digital libraries, Jeng ${ }^{33,34}$ found that ease of use, satisfaction, efficiency and effectiveness are the main applied criteria. Some of the evaluation studies extend to assess performance, content and services of digital libraries while service evaluation mainly concentrates on digital reference. ${ }^{35}$ Other evaluation studies also look into the impact of digital libraries. ${ }^{10}$

Little research has investigated user's evaluation of digital libraries, in particular, their criteria and their actual assessment of digital libraries. ${ }^{9,36}$ However, as the success of a digital library depends on the users, the value of digital libraries needs be judged by the users of digital libraries. Therefore, in this paper, we present a model based on fuzzy linguistic information to evaluate the quality of digital libraries which is defined using users' perceptions on the quality of digital services provided through their Web site.

\subsection{A model based on fuzzy linguistic information to evaluate the quality of digital libraries}

We use the information quality framework ${ }^{37}$ defined in the context of management information systems as basis of our model to evaluate the quality of digital libraries. It has been satisfactorily applied to previous quality models for personal Web sites, ${ }^{38}$ mobile Internet services ${ }^{39}$ and Web sites that store Web documents. ${ }^{40,41}$ In this information quality framework is established that the quality of the information systems cannot be assessed independently of the information consumers' 
opinions (people who use information). This framework defines four major quality dimensions: ${ }^{37}$

(i) Intrinsic quality. This dimension addresses the very nature of the information. It assumes that information has its own quality. The main criterion of the intrinsic quality is the accuracy of the information. If a reputation for inaccurate information becomes common knowledge for a particular information system, this system is viewed as having little added value and will result in a reduction of use. Other criteria of this dimension are: believability, reputation and objectivity.

(ii) Contextual quality. This dimension emphasizes the importance of the informative aspects of information but from a task perspective. It highlights the requirement that information quality must be considered within the context of the task in hand; it must be relevant, timely, complete, and appropriate in terms of amount, so as to add value to the tasks for which the information is provided. Therefore, some criteria of this dimension are: value-added, relevance, completeness, timeliness, and appropriate amount.

(iii) Representational quality. This dimension emphasizes the importance of the technical aspects of the (computer-based) structure of the information. It requires information systems to present their information in such a way that it is interpretable, easy to understand, easy to manipulate, and is represented concisely and consistently. Therefore, some of its criteria are: understandability, interpretability, concise representation, and consistent representation.

(iv) Accessibility quality. This dimension emphasizes the importance of the technical aspects of computer systems that provided access to information. It requires the information system to be accessible but secure. Therefore, among the criteria of this dimension are: accessibility and secure access.

We adapt this information quality framework to develop our evaluation model of the quality of digital libraries. However, as it is oriented to users because the user participation in the quality evaluation processes of services is fundamental to correctly draw the situation of the service, we are going to define a low number of subjective criteria being easily understandable by the users in order to they do not cause the rejection of the users.

Taking into account these considerations, we define a model to evaluate the quality of digital libraries focused on digital services provided through their Web sites. This model presents two elements: (i) a evaluation scheme that contains the subjective criteria and (ii) a computation method to generate quality assessments of digital libraries.

\subsubsection{Evaluation scheme to characterize the quality in digital libraries}

According to the quality framework, $37,38,39,40$ we develop an evaluation scheme for evaluating the quality of digital libraries. This evaluation scheme is based both on 
technical criteria of digital libraries design and on criteria related to the content of information of digital libraries. These criteria are assessed subjectively by users who occasionally visit the digital libraries because they find something that satisfies their information needs.

The evaluation scheme proposed presents the following characteristics:

(i) It is user driven rather than designed driven. We want to evaluate the quality of digital libraries from the evaluations provided by the different users of them. Therefore, the evaluation scheme should be user driven rather than designed driven from two perspectives:

(a) Qualitative perspective: The evaluation scheme necessarily requires the inclusion of criteria easily understandable to any user (e.g., relevance, understandability) rather than criteria that can be measured objectively independently of users (ratio of digital journals) or only perceptible by the designers (e.g., code quality or design).

(b) Quantitative perspective: The evaluation scheme should not include an excessive number of quality criteria in order to help users in understanding it and avoiding confusion. Furthermore, long and complex evaluation schemes cause user idleness and limit their own application possibilities.

(ii) It is weighted: i.e., its quality criteria are not equally important. The quality criteria of the evaluation scheme do not play equal roles in measuring the information quality of a digital library: i.e., some criteria should be more influential than others. For example, user opinions on the information quality of digital libraries (e.g., coverage of the digital library about search topics) must be an important criterion of the evaluation scheme.

We define a user driven and weighted evaluation scheme of digital libraries that contemplates the following four quality dimensions together with their digital quality criteria:

(i) Intrinsic quality of digital libraries: To evaluate the intrinsic quality or accuracy of digital libraries, we define the following subjective criterion: you find what you are looking for.

(ii) Contextual quality of digital libraries: To evaluate the information quality of the digital libraries within the context, the following subjective criteria are defined: coverage of the digital library about search topics, information electronic services about new inputs, added value information profits and also global satisfaction degree.

(iii) Representational quality of digital libraries: It is evaluated taking into account the following subjective criteria: understandability of the digital library Web site and training received.

(iv) Accessibility and interaction quality of digital libraries: It is measured considering the following subjective criteria: variety of search tools, navigability of the 
digital library Web site, satisfaction degree with the computing infrastructure and satisfaction degree with the response time.

\subsubsection{Computation method to generate quality assessments in digital libraries}

The computation method to generate quality assessments in digital libraries is like a multi-person multi-criteria decision making method in which the search alternatives are digital libraries. In a multi-criteria decision making method, the goal consists of searching the best alternatives according to the assessments provided by a group of experts with respect to a set of evaluation criteria. ${ }^{42}$ To do that, through the aggregation of the experts' assessments the quality of alternatives is measured and, later, the exploitation of those quality values leads to the selection of the best alternatives. In our case, the goal consists of computing quality evaluations of digital libraries in order to select the digital library that could better meet the user information needs, but as in a multi-criteria decision context, we compute those values according to the assessments provided by a group of persons (digital libraries users).

As it is known, in multi-criteria decision making processes the chosen aggregation operator is a critical aspect that has a direct influence on the success of the decision process. The quantifier guided aggregation operators based on the OWA operator constitute a successful tool to aggregate information because of its flexibility: i.e., it allows representation in the aggregations of different interpretations of the concept of majority by means of the fuzzy linguistic quantifier. ${ }^{27}$ We do the same in our computation method.

We have designed a computation method to generate quality assessment in digital libraries that has two main characteristics:

(i) It is a user centered computation method. The quality assessment in digital libraries is obtained from individual linguistic judgments provided by digital libraries users rather than from assessments obtained objectively by means of the direct observation of the digital libraries characteristics.

(ii) It is a majority guided computation method. The quality assessments are values representative of the majority of individual judgments provided by the digital libraries users. The aggregation to compute the quality assessments is developed by means of the LOWA and LWA operators.

Firstly, we define a quality evaluation questionnaire providing questions for each one of the subjective criteria proposed in the evaluation scheme, i.e., there are eleven questions: $\left\{q_{1}, \ldots, q_{11}\right\}$. For example, for the subjective criterion you find what you are looking for, the question $q_{1}$ can be: "What is the degree in which you usually find what you are looking for?". The quality evaluation questionnaire can be as follows: 


\section{QUALITY EVALUATION QUESTIONNAIRE}

Question 1: "What is the degree in which you usually find what you are looking for?"

Question 2: "What is the coverage degree of the digital library about search topics?"

Question 3: "What is the degree of information electronic service about new inputs?"

Question 4: "What is the degree of added value information profits?"

Question 5: "What is your global satisfaction degree?"

Question 6: "What is the understandability degree of the digital library Web site?"

Question 7: "What is the degree of training received?"

Question 8: "What is the degree of variety of search tools?"

Question 9: "What is the navigability degree of the digital library Web site?"

Question 10: "What is your satisfaction degree with the computing infrastructure?"

Question 11: "What is your satisfaction degree with the response time?"

The concept behind each question is rated on a linguistic term set $S$. To do so, we can use the set of linguistic terms proposed in Sec. 2 to rate all the questions. We use fuzzy linguistic variables to represent users' opinions by mean of linguistic labels because they are more easily understood by the users than numerical ones. In addition, we assume that each subjective criteria does not have the same importance in the evaluation scheme, i.e., it is assigned a relative linguistic importance degree for each subjective criterion: $\left\{I\left(q_{1}\right), \ldots, I\left(q_{11}\right)\right\}, I\left(q_{i}\right) \in S$. These importance degree could be obtained from a set of experts or users' judgements. ${ }^{43}$

Then, assuming that we have a group of users, $\left\{e_{1}, \ldots, e_{L}\right\}$, that have filled in the questionnaire, and given a digital library, $\mathcal{A}_{m}$, the computation method generates its quality assessment, $r^{m} \in S$, using the linguistic aggregation operators LOWA and LWA in the following steps:

(i) Calculate for each subjective criterion, $q_{i}$, the global quality assessment, $r_{i}^{m} \in S$, by aggregating the evaluation judgments provided by the group of users on the subjective criterion by means of the LOWA operator $\phi$ :

$$
r_{i}^{m}=\phi_{Q}\left(e_{1}\left(q_{i}\right), \ldots, e_{L}\left(q_{i}\right)\right),
$$

where $e_{l}\left(q_{i}\right) \in S$ is the linguistic preference provided by the $e_{l}$ on subjective criteria represented by the question $q_{i}$. Therefore, $r_{i}^{m}$ is a linguistic measure that represents the quality assessment of the digital library $\mathcal{A}_{m}$ with respect to subjective criterion $q_{i}$ according to the majority (represented by the fuzzy linguistic quantifier $Q$ ) of linguistic evaluation judgments provided by the group of users $\left\{e_{1}, \ldots, e_{L}\right\}$. 
(ii) Calculate for the digital library, $\mathcal{A}_{m}$, its quality assessment, $r^{m} \in S$, by aggregating its individual quality assessment, $r_{i}^{m} \in S$, for each subjective criterion, $q_{i}$, by means of the LWA operator $\Phi$ :

$$
r^{m}=\Phi_{Q}\left(\left(I\left(q_{1}\right), r_{1}^{m}\right), \ldots,\left(I\left(q_{11}\right), r_{11}^{m}\right)\right) .
$$

In this case, $r^{m}$ is a measure that represents the quality assessment of the digital library $\mathcal{A}_{m}$ according to the majority (represented by the fuzzy linguistic quantifier $Q$ ) of linguistic evaluation judgments provided by the group of users about important subjective criteria $q_{i}$.

\section{Example of Application}

In this section, we present an example where the evaluation model is applied to evaluate the quality of three Spanish academic digital libraries:

(i) Digital library of Jaén University.

(ii) Digital library of Córdoba University.

(iii) Digital library of Málaga University.

The reason for the selection of academic digital libraries is due to they are the first kind of libraries that are getting most benefit from the Web possibilities to help in teaching, learning and researching activities. ${ }^{2,44,45}$

Fifty subjects were recruited for this study. They were $\mathrm{Ph}$. D. students from University of Granada who were interested in the learning and using of digital libraries. Female (56\%) and male (44\%) subjects are pretty close in the composition of the subject pool. All of them had enough knowledge of digital libraries, and have used and searched digital libraries before this study. Two reasons were considered for the recruitment:

(i) These subjects have a need to understand digital libraries and have some experience with the use of digital libraries.

(ii) These subjects are the targeted audience for similar types of digital libraries. They are users of the digital library of Granada University.

The best way to evaluate digital libraries is to actually use them. Fifty subjects tried to find information related to six questions for each one of the three academic digital libraries selected for this study. For example, subjects were instructed to find a book titled "Fuzzy set theory and its applications" and its authors. In another question, subjects need to identify two approaches to find information about L. A. Zadeh and five papers of him. The subjects could work on the digital libraries at any locations that they felt comfortable.

To obtain the judgements supplied by the users for each academic digital library, we use the quality evaluation questionnaire presented in Sec. 3. It is composed of the eleven queries, one for each subjective criterion $q_{i}$. Furthermore, the set of nine labels proposed in Sec. 2 is used to provide the user evaluations. In Table 1, we 
can see the global quality assessment, $r_{i}^{m}=\phi_{Q}\left(e_{1}\left(q_{i}\right), \ldots, e_{50}\left(q_{i}\right)\right) \in S$, for each subjective criterion $q_{i}$ for each academic digital library selected for this study.

Table 1. Global quality assessment, $r_{i}^{m} \in S$, for each subjective criterion $q_{i}$.

\begin{tabular}{cccccccccccc}
\hline & $q_{1}$ & $q_{2}$ & $q_{3}$ & $q_{4}$ & $q_{5}$ & $q_{6}$ & $q_{7}$ & $q_{8}$ & $q_{9}$ & $q_{10}$ & $q_{11}$ \\
\hline Jaén & $V H$ & $V H$ & $L$ & $M$ & $V H$ & $V H$ & $M$ & $H$ & $E H$ & $E H$ & $V H$ \\
Málaga & $E H$ & $E H$ & $L$ & $M$ & $V H$ & $V H$ & $H$ & $M$ & $V H$ & $E H$ & $H$ \\
Córdoba & $V H$ & $H$ & $L$ & $M$ & $H$ & $V H$ & $M$ & $H$ & $M$ & $V H$ & $H$ \\
\hline
\end{tabular}

In this particular example, we can see that the subjective criterion $q_{3}$ has a lower evaluation in the three academic digital libraries, that is, users think that the information electronic services about new inputs should be improved. In addition, the subjective criterion $q_{6}$ has a higher evaluation in the three academic digital libraries, in fact, its evaluation is near to the maximum one, which means that almost every user agrees on the good evaluation of the understandability degree of the three digital libraries Web sites studied. On the other hand, subjective criteria $q_{9}$ has a much better evaluation for the academic digital library of Jaén than for the academic digital library of Córdoba, which means that the navigability of the digital library Web site should be improved in the academic digital library of Córdoba.

Assuming the linguistic importance degrees $\{E H, E H, M, M, T, H, M, M, H, M, M\}$ associated with the quality criteria, the linguistic quantifier most of defined as $Q(r)=r^{1 / 2}$, and using the LWA operator, in Table 2 , we can see the quality assessment, $r^{m}=\Phi_{Q}\left(\left(I\left(q_{1}\right), r_{1}^{m}\right), \ldots,\left(I\left(q_{11}\right), r_{11}^{m}\right)\right) \in S$, for each academic digital library selected for this study.

Table 2. Quality assessment, $r^{m} \in S$, for each academic digital library.

\begin{tabular}{cccc}
\hline & Jaén & Málaga & Córdoba \\
\hline$r^{m}$ & $H$ & $H$ & $M$ \\
\hline
\end{tabular}

For example, the quality assessment for the academic digital library of Jaén is obtained from the following expression:

$$
\begin{aligned}
& r^{J a e n}=\Phi_{Q}((E H, V H),(E H, V H),(M, L),(M, M),(T, V H),(H, V H),(M, M), \\
& (M, H),(H, E H),(M, E H),(M, V H))=H .
\end{aligned}
$$

To develop this expression it is necessary to calculate the weighting vector $W$. To do so, we make use of the linguistic quantifier most of defined as $Q(r)=r^{1 / 2}$ :

$$
Q(0)=0, \quad Q(1 / 11)=0.30, \quad Q(2 / 11)=0.43, \quad Q(3 / 11)=0.52
$$




$$
\begin{gathered}
Q(4 / 11)=0.60, \quad Q(5 / 11)=0.67, \quad Q(6 / 11)=0.74, \quad Q(7 / 11)=0.80 \\
Q(8 / 11)=0.85, \quad Q(9 / 11)=0.90, \quad Q(10 / 11)=0.95, \quad Q(1)=1
\end{gathered}
$$

And, using Eq. (2.3), we obtain the following weighting vector $W$ :

$$
W=(0.30,0.13,0.09,0.08,0.07,0.07,0.06,0.05,0.05,0.05,0.05)
$$

As orness $(W)=0.67 \geq 0.5$, then $h=M I N\left(c_{i}, a_{i}\right)$, and therefore:

$$
\begin{aligned}
& \Phi_{Q}((E H, V H),(E H, V H),(M, L),(M, M),(T, V H),(H, V H),(M, M),(M, H), \\
& (H, E H),(M, E H),(M, V H))=\phi_{Q}(V H, V H, L, M, V H, H, M, M, H, M, M)= \\
& W \cdot B^{T}=C^{11}((V H, 0.30),(V H, 0.13),(V H, 0.09),(H, 0.08),(H, 0.07),(M, 0.07), \\
& (M, 0.06),(M, 0.05),(M, 0.05),(M, 0.05),(L, 0.05))=0.30 \odot V H \oplus 0.70 \odot \\
& C^{10}((V H, 0.19),(V H, 0.13),(H, 0.11),(H, 0.10),(M, 0.10),(M, 0.08),(M, 0.07), \\
& (M, 0.07),(M, 0.07),(L, 0.07))=H .
\end{aligned}
$$

\section{Discussion}

In this section, we analyze some possible drawbacks and advantages of the model presented in this paper to evaluate the quality of digital libraries. We also outline some possible improvements.

- Drawbacks. The main drawback of the proposed evaluation model is that it is strongly dependent on the degree to which users decide to participate by providing their opinions. The problem with asking people for the quality dimensions is that the cost, in terms of time and effort, of providing linguistic evaluation judgments generally outweighs the reward people will eventually receive. To provide evaluation judgments requires selflessness in users because the judgments provided will only help to the improvement of the digital library. How users should be compensated for offering their opinions on quality criteria is the question. This is a common problem with other Web technologies in which user participation is necessary, for example, recommender systems. ${ }^{46,47}$

- Advantages. The main advantage of our evaluation model is that it is designed to facilitate user participation. Many Web quality evaluation approaches ${ }^{48,49}$ assume that user perceptions are necessary to measure the quality of Web sites, but they do not provide enough means to facilitate user participation and to represent and adequately exploit user evaluation judgments. In our evaluation model, the user driven evaluation scheme facilitates user participation, the fuzzy linguistic modeling is a good tool to represent user evaluation judgments, and the linguistic aggregation operators allow adequate use of the user evaluation judgments in order to generate the quality assessments of digital libraries ${ }^{50}$.

- Improvements. The proposed evaluation model may be extended to include additional tools to improve the quality of the linguistic evaluations generated. In our proposal, we assume that digital libraries users know perfectly the meaning of the linguistic scales used to provide the linguistic evaluation judgments that 
are expressed in English. However, this is not a realistic assumption because the World Wide Web is a multilingual tool. Therefore, including in our model the possibility of using multilingual linguistic scales could increase the user collaboration in our evaluation model. A possible approach could consist of the use of multi-granular fuzzy linguistic modeling ${ }^{51}$ to represent the linguistic evaluation judgments expressed in different languages.

\section{Concluding Remarks}

The development of digital libraries is to satisfy user need. Therefore, user satisfaction is essential for the success of a digital library. In this way, we have presented a model based on fuzzy linguistic information to evaluate the quality of digital libraries, which is defined using users' perceptions on the quality of digital services provided through their Web sites. The evaluation model is composed of two components, a user driven evaluation scheme and a user centered computation method. Therefore, this model is user oriented because it only considers user evaluation judgments to evaluate the quality of digital libraries. To do so, we have assumed a fuzzy linguistic modeling to represent the users' perceptions because it is more easily understood by the users than numerical one. Considerable use is made of fuzzy set technology to provide the ability to describe the information by using linguistic label in a way that is particularly user friendly. Furthermore, we have applied automatic tools of fuzzy computing with words based on the LOWA and LWA operators to compute global quality assessments of digital libraries.

In the future, we propose to continue this research approach in several directions:

(i) To improve the evaluation of digital libraries by incorporating information on users that supply the evaluation judgments of the digital libraries, e.g., their levels of expertise in the topic (specialists, knowledgeable, inexperienced people).

(ii) To implement a recommender system ${ }^{47}$ that incorporates the generation procedure of recommendations for improving the digital services provided by the digital libraries through their Web sites using the quality assessments provided by the users.

(iii) To redefine the evaluation model of digital libraries to create a feedback mechanism that can be used to improve such design aspects, as information content aspects of the digital library, by mining the users' opinions by means of appropriate mining tools. ${ }^{52}$

\section{Acknowledgments}

This paper has been developed with the Financing of FEDER funds in FUZZYLING project (TIN2007-61079), PETRI project (PET2007-0460), Andalucian Excellence project (TIC-5299) and project of Ministry of Public Works (90/07). 


\section{References}

1. S. Lawrence and C. L. Giles, Searching the World Wide Web, Science 280(5360) (1998) 98-100.

2. W. Y. Arms, Digital Libraries (MIT Press, Cambridge, 2001).

3. D. Bawden and P. Vilar, Digital libraries: To meet or manage user expectations, Aslib Proceedings: New Information Perspectives 58(4) (2006) 346-354.

4. G. G. Chowdhury and S. Chowdhury, Introduction to Digital Libraries (Facet Publisher, London, 2003).

5. J. M. Morales-del-Castillo, R. Pedraza-Jiménez, A. A. Ruíz, E. Peis and E. HerreraViedma, A semantic model of selective dissemination of information for digital libraries, Information Technology and Libraries 28(1) (2009) 22-31.

6. C. Rushbridge, Towards the hybrid library, D-Lib Magazine July/August (1998).

7. M. E. Renda and U. Straccia, A personalized collaborative digital library environment: A model and an application, Information Processing and Management 41(1) (2005) $5-21$.

8. N. Fuhr, G. Tsakonas, T. Aalberg, M. Agosti, P. Hansen, S. Kapidakis, et al., Evaluation of digital libraries, International Journal on Digital Libraries 8(1) (2007) 21-38.

9. H. I. Xie, Users' evaluation of digital libraries (DLs): Their uses, their criteria, and their assessment, Information Processing and Management 44(3) (2008) 1346-1373.

10. G. Marchionini, Evaluation digital libraries: A longitudinal and multifaceted view, Library Trends 49(2) (2000) 304-333.

11. S. H. Tsaur, T. Y. Chang and C. H. Yen, The evaluation of airline service quality by fuzzy MCDM, Tourism Management 23(2) (2002) 107-115.

12. F. Herrera, E. Herrera-Viedma and J. L. Verdegay, Direct approach processes in group decision making using linguistic OWA operators, Fuzzy Sets and Systems 79(2) (1996) 175-190.

13. F. Herrera and E. Herrera-Viedma, Aggregation operators for linguistic weighted information, IEEE Transactions on Systems, Man and Cybernetycs, Part A: Systems and Humans 27(5) (1997) 646-656.

14. S. Alonso, E. Herrera-Viedma, F. Chiclana and F. Herrera, Individual and social strategies to deal with ignorance situations in multi-person decision making, International Journal of Information Technology \& Decision Making 8(2) (2009) 313-333.

15. Y. Gao, G. Zhang and J. Lu, A fuzzy multi-objective bilevel decision support system, International Journal of Information Technology \& Decision Making 8(1) (2009) 93108.

16. A. P. Tchangani, Evaluation model for multiattributes-multiagents decision making: Satisfacing game approach, International Journal of Information Technology \& Decision Making 8(1) (2009) 73-91.

17. C. B. Ho, D. D. Wu and D. L. Olson, A risk scoring model and application to measuring internet stock performance, International Journal of Information Technology \& Decision Making 8(1) (2009) 133-149.

18. E. Herrera-Viedma, An information retrieval system with ordinal linguistic weighted queries based on two weighting elements, International Journal Uncertainty, Fuzziness and Knowledge-Based Systems 9(1) (2001) 77-88.

19. E. Herrera-Viedma and E. Peis, Evaluating the informative quality of documents in SGML-format using fuzzy linguistic techniques based on computing with words, Information Processing and Management 39(2) (2003) 233-249.

20. V. Loia, W. Pedrycz, S. Senatore and M. I. Sessa, Web navigation support by means of cognitive proximity-driven assistant agents, Journal of the American Society for Information Science and Technology 57(4) (2006) 512-527. 
21. E. Herrera-Viedma, A. G. López-Herrera, M. Luque and C. Porcel, A fuzzy linguistic IRS model based on a 2-tuple fuzzy linguistic approach, International Journal of Uncertainty, Fuzziness and Knowledge-based Systems 15(2) (2007) 225-250.

22. F. J. Cabrerizo, S. Alonso and E. Herrera-Viedma, A consensus model for group decision making problems with unbalanced fuzzy linguistic information, International Journal of Information Technology \& Decision Making 8(1) (2009) 109-131.

23. L. A. Zadeh, The concept of a linguistic variable and its applications to approximate reasoning. Part I, Information Sciences 8(3) (1975) 199-249.

24. L. A. Zadeh, The concept of a linguistic variable and its applications to approximate reasoning. Part II, Information Sciences 8(4) (1975) 301-357.

25. L. A. Zadeh, The concept of a linguistic variable and its applications to approximate reasoning. Part III, Information Sciences 9(1) (1975) 43-80.

26. G. A. Miller, The magical number seven or minus two: Some limits on our capacity of processing information, Psychological Review 63(2) (1956) 81-97.

27. R. R. Yager, On ordered weighted averaging aggregation operators in multicriteria decision making, IEEE Transactions on Systems, Man and Cybernetycs 18(1) (1988) $183-190$.

28. J. Kacprzyk, Group decision making with a fuzzy linguistic majority, Fuzzy Sets Systems 18(2) (1986) 105-118.

29. L. A. Zadeh, A computational approach to fuzzy quantifiers in natural languages, Computer \& Mathematics with Applications 9(1) (1983) 149-184.

30. R. R. Yager, A note on weighted queries in information retrieval systems, Journal of the American Society for Information Science 38(1) (1987) 23-24.

31. T. Saracevic, Digital library evaluation: Toward an evolution of concepts, Library Trends 49(3) (2000) 350-369.

32. T. Saracevic and L. Covi, Challenges for digital library evaluation, Proceedings of the American Society for Information Science 37 (2000) 341-350.

33. J. Jeng, Usability assessment of academic digital libraries: Effectiveness, efficiency, satisfaction, and learnability, LIBRI 55(2-3) (2005) 96-121.

34. J. Jeng, What is usability in the context of the digital library and how can it be measured?, Information Technology and Libraries 24(2) (2005) 47-56.

35. D. S. Carter and J. Janes, Unobtrusive data analysis of digital reference questions and service at the Internet Public Library: An exploratory study, Library Trends 49(2) (2000) 251-265.

36. H. I. Xie, Evaluation of digital libraries: Criteria and problems from user's perspectives, Library and Information Science Research 28(3) (2006) 433-452.

37. K. Huang, Y. W. Lee and R. Y. Wang, Quality Information and Knowledge (Prentice Hall, Upper Saddle River, NJ, 1999).

38. P. Katerattanakul and K. Siau, Measuring information quality of web sites: Development of an instrument, in Proc. of 20th Int. Conf. on Inf. Sys., (Charlotte, 1999), pp. 279-285.

39. M. Chae and J. Kim, Quality for mobile internet devices: A theoretical model with empirical validation, Electronic Markets 12(1) (2002) 38-46.

40. E. Herrera-Viedma, G. Pasi, A. G. López-Herrera and C. Porcel, Evaluating the information quality of web sites: A methodology based on fuzzy computing with words, Journal of American Society for Information Science and Technology 57(4) (2006) $538-549$.

41. E. Herrera-Viedma, E. Peis, J. M. Morales-del-Castillo, S. Alonso and E. K. Anaya, A fuzzy linguistic model to evaluate the quality of web sites that store XML documents, International Journal of Approximate Reasoning 46 (2007) 226-253. 
42. M. Köksalan and C. Tuncer, A dea-based approach to ranking multi-criteria alternative, International Journal of Information Technology \& Decision Making 8(1) (2009) 29-54.

43. P. Zhang and G. Von Dran, Expectations and rankings of websites quality features: results of two studies on user perception, in Proc. of the 34th Hawaii Inter. Conference on System Sciences, (Hawaii, 2001), pp. 1-9.

44. G. Marchionini and H. Maurer, The roles of digital libraries in teaching and learning, Communication of ACM 38(4) (1995) 67-75.

45. S. R. Sharifabadi, How digital libraries can support e-learning, The Electronic Library 24(3) (2006) 389-401.

46. P. Raghavan, Social networks and the Web, Lecture Notes in Artificial Intelligence 3034 (2004) 1-1.

47. C. Porcel, A. G. López-Herrera and E. Herrera-Viedma, A recommender system for research resources based on fuzzy linguistic modeling, Expert Systems with Applications, 36 (2009) 5173-5183.

48. A. M. Aladwani and P. C. Palvia, Developing and validating an instrument for measuring user perceived Web quality, Information \& Management 39(6) (2002) 467-476.

49. L. Mich, M. Franch and L. Gaio, Evaluating and designing Web site quality, IEEE Multimedia 10(1) (2003) 34-43.

50. E. Herrera-Viedma, J. López-Gijón, S. Alonso, J. Vilchez, C. García, L. Villén and A. G. López-Herrera, Applying aggregation operators for information access systems: An application on spanish digital libraries, Internacional Journal of Intelligent Systems 23(12) (2008) 1235-1250.

51. F. Mata, L. Martínez and E. Herrera-Viedma, An adaptive consensus support model for group decision making problems in a multi-granular fuzzy linguistic context, IEEE Transactions on Fuzzy Systems 17(2) (2009) 279-290.

52. Y. Peng, G. Kou, Y. Shi and Z. Chen, A descriptive framework for the field of data mining and knowledge discovery, International Journal of Information Technology \& Decision Making 7(4) (2008) 639-682. 